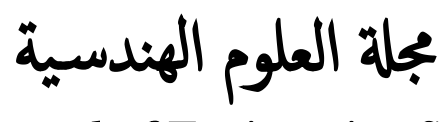

\title{
Investigation of General Solution in Free Datum Networks
}

\author{
Mudathir Omer Ahmed 1,* \\ ${ }^{1}$ Surveying Engineering Department University- Faculty of Engineering Sciences, Omdurman Islamic University. \\ *Corresponding author: Mudathir O. A. (e-mail: mudathiro@yahoo.com).
}

Article history: Received 16 February 2019, Received in revised form 04 May 2019, Accepted 16 May 2019

\begin{abstract}
The datum of survey control networks sometimes cannot be defined because there is no position of fixed points is provided. In these types of networks the solution can be obtained by what is known as general solution of free datum networks. Actually, the datum in free networks is defined by additional datum constraints to solve the problem of singularity of the normal equations matrix. To solve the problem of datum defect, the unknown parameters of the datum are required to be fixed. These constraints can be established for a part or all points of the network to define the reference frame of the control network in several ways. The adjustment obtained using full datum constraints is called free or relative datum adjustment. In this research the various forms of datum constraints are established and investigated in order to distinguish between them. More than one solution models applied individually as different cases to be compared with others. Conclusions drawn that the optimum solution with minimum norm and minimum trace is that the general solution using full constraints matrix, it gives the same coordinate differences obtained by others. Then it can be widely used in solution of survey control networks used in many types of networks such as these applied in deformation monitoring field. Other conclusion is that the suitability of the general solution form for all other chosen datums. Any fixed datum solution can be obtained from the general solution by adding a scalar calculated from height(s) of the reference fixed point(s).
\end{abstract}

Keywords: Datum constraints, free datum, general solution, minimum trace, minimum norm, network.

\section{INTRODUCTION}

Usually, in survey control networks the datum is defined by fixing its parameters which is done by establishing a constraint for each parameter. The number of unknown parameters is equal to the number of datum defects of the network to be adjusted and it depends on the type of the network.

In one dimensional networks (leveling or gravity networks) the datum defect is equal to one therefore the datum is defined by fixing one parameter. In plane two dimensional (horizontal) control networks the datum is defined four parameters, two of them defining the origin of the network, one for orientation and one for scale factor. Alternatively, the four parameters can be defined completely by fixing the position of two points on the earth surface. In three dimensional networks the datum is defined by the fixation of seven parameters, three for position, three for orientation and one for scale.

The number of datum parameters (defects) in survey control networks can be summarized in Table 1.

A datum constraint for each parameter is required to solve the problem of singularity in the normal matrix of solution. Starting from the well-known functional relationship

$$
A_{n \times m} x_{m \times 1}=b_{n \times 1}+v_{n \times 1}
$$

The least squares estimate of the parameters, $\hat{x}$, is given from the following normal equation matrix: $\left(A^{T} W A\right) \hat{x}=A^{T} W b$ i.e. $\quad N \hat{x}=U$ 
The solution of the model in (2) can easily be obtained for a fixed-datum using the usual Caley inverse. However, in the case of free datum networks the solution cannot be obtained directly due to the defect of the normal equation caused by the unknown datum parameters. The defect of the normal matrix comes from the defect of design matrix A can be obtained from:

$\mathrm{d}=\operatorname{dim}(\mathrm{A})-\mathrm{r}(\mathrm{A})$

where (dim) is the number of columns and (r) is the rank (number of linearly independent columns) as defined by [6].

In the case of the free datum networks the columns of the design matrix A are linearly dependent i.e. some of its columns are linearly related to others, and also those of the normal matrix $\mathrm{N}$.

If a two dimensional network is taken, four parameters are required to be defined. This is done by using one constraint for each parameter then, four datum constraints are required to be established as follows.

Two constraints to define the origin of the network by fixing the position of one point e.g the centroid of the network:

$\frac{1}{n} \sum x_{i}=$ const.

$\frac{n}{1} \sum x_{i}=$ const. $\quad \sum d x_{i}=0$

$\frac{1}{n} \sum y_{i}=$ const. $\quad$ or $\quad \sum d y_{i}=0$.

$\left[\begin{array}{ccc}1 & 0 & \ldots . . . . \\ 0 & 1 & \ldots . . . . .\end{array}\right]\left[\begin{array}{c}d x_{1} \\ d y_{1} \\ \vdots\end{array}\right]=\left[\begin{array}{c}0 \\ 0 \\ \vdots\end{array}\right]$

One constraint to define the orientation of the network e.g. the mean bearing from the centroid of the network:

$$
\begin{array}{r}
\frac{1}{n} \sum \alpha_{i}=\text { const. } \quad \text { or } \sum d \alpha_{i}=0 \\
{\left[\begin{array}{ccc}
\bar{y} & -\bar{x} & \ldots . . . . . . .
\end{array}\right]\left[\begin{array}{c}
d x_{1} \\
d y_{1} \\
\vdots
\end{array}\right]=\left[\begin{array}{c}
0 \\
0 \\
\vdots
\end{array}\right]}
\end{array}
$$

One constraint to define the scale of the network e.g. the mean distance from the centroid of the network:

$$
\frac{1}{n} \sum \ell_{i o}=\text { const. } \quad \text { or } \quad \sum d \ell_{i o}=0
$$

$\left[\begin{array}{lll}\bar{x} & \bar{y} & \ldots \ldots \ldots . . .\end{array}\right]\left[\begin{array}{c}d x_{1} \\ d y_{1} \\ \vdots\end{array}\right]=\left[\begin{array}{c}\mathrm{o} \\ \mathrm{o} \\ \vdots\end{array}\right]$

where $\bar{x}$ and $\bar{y}$ are the approximate coordinates reduced to the centroid of the network.

The models in (4), (5) and (6) are applied to some or all points of the network to define the datum definition. Then, the general equation for the datum constraints is given by:

$D^{T} x=0$

where D: is the coefficients matrix of the datum constraints.

Table 1. Datum Parameters of Survey Control networks

\begin{tabular}{|l|l|l|}
\hline Network & $\begin{array}{l}\text { No. of } \\
\text { defects }\end{array}$ & $\begin{array}{l}\text { Type of } \\
\text { parameters }\end{array}$ \\
\hline Vertical networks & 1 & 1-translation \\
\hline Horizontal networks & 4 & $\begin{array}{l}\text { 2-translations } \\
\text { 1-rotaion } \\
\text { 1-scale factor }\end{array}$ \\
\hline $\begin{array}{l}\text { Three-dimensional } \\
\text { networks }\end{array}$ & 7 & $\begin{array}{l}\text { 3-translations } \\
\text { 3-rotations } \\
\text { scale factor }\end{array}$ \\
\hline
\end{tabular}

\section{COEFFICIENTS MATRIX FOR FULL DATUM CONSTRAINTS}

The coefficients matrix (D) constructed using all points of the network called the full datum constraints and denoted by (S). To establish this matrix starting from the idea that it has column vectors generate the null space of the matrix $\mathrm{A}$, then matrix relation can be given as [2]:

AS $=0$

On the other hand, the normal matrix $\mathrm{N}$ shares the same null space as the matrix A therefore, $\mathrm{NS}=\mathrm{ATWAS}=0$

Firstly, the design matrix A can be partitioned in order to column dependency as:

$\mathrm{A}=\left[A_{1} \vdots A_{2}\right]$ where $\mathrm{A}_{1}$ has $\mathrm{r}$ (No.of rank) independent columns and $\mathrm{A} 2$ has the remaining $\mathrm{d}$ (No. of defect) columns.

Using identity matrices $\mathrm{I}_{\mathrm{r}}$ and $\mathrm{I}_{\mathrm{d}}$ of dimensions equal to $\mathrm{rxr}$ and $\mathrm{dxd}$ respectively to rebuild $\mathrm{A}_{1}$ and $\mathrm{A}_{2}$ as follows: 


$$
A_{1}=A\left[\begin{array}{c}
I_{r} \\
0
\end{array}\right], A_{2}=A\left[\begin{array}{c}
0 \\
I_{d}
\end{array}\right]
$$

The linear dependency between A1 and A2 means that there is a matrix $\mathrm{L}$ gives the following relation $[3,4,6]$.

$$
\mathrm{A}_{1} \mathrm{~L}=\mathrm{A}_{2}
$$

then:

$$
\begin{gathered}
A\left[\begin{array}{c}
\boldsymbol{I}_{r} \\
\mathbf{O}
\end{array}\right] \boldsymbol{L}=A\left[\begin{array}{c}
\mathrm{O} \\
\boldsymbol{I}_{d}
\end{array}\right] \\
A\left[\begin{array}{c}
\boldsymbol{L} \\
\mathbf{O}
\end{array}\right]=A\left[\begin{array}{c}
\mathbf{O} \\
\boldsymbol{I}_{d}
\end{array}\right] \Rightarrow A\left[\begin{array}{l}
\boldsymbol{L} \\
\mathbf{O}
\end{array}\right]-A\left[\begin{array}{c}
\mathbf{O} \\
\boldsymbol{I}_{d}
\end{array}\right]=\mathbf{O}=A\left[\begin{array}{c}
\boldsymbol{L} \\
-\boldsymbol{I}_{d}
\end{array}\right]
\end{gathered}
$$

In order to satisfy Eq (8), $\mathrm{S}$ can be selected as follows:

$$
S=\left[\begin{array}{c}
L \\
-I_{d}
\end{array}\right]
$$

The matrix $\mathrm{L}$ can be derived from $\mathrm{Eq}(11)$ as follows:

$L=\left(A_{1}^{T} A_{1}\right)^{-1} A_{1}^{T} A_{2}$

However, the matrix $S$ usually expresses the datum defect in the free network. For fully defective networks this matrix can be constructed as follows [2]:

- In the case of the vertical networks, the matrix $S$ is a unit vector with dimensions $(\mathrm{p} X 1)$ i.e. $\mathrm{ST}=\left[\begin{array}{lll}1 & 1 & 1 \ldots \ldots .1\end{array}\right]^{\mathrm{T}}$

where $\mathrm{p}$ is the number of unknown points.

- In the case of two dimensional networks, the matrix $S$ has dimensions $(2 \mathrm{p} \times 4)$ and is given as follows:

$$
\begin{aligned}
S^{T} & =\left[\begin{array}{cccc}
1 & 0 & 1 & 0 \ldots \ldots \ldots \\
0 & 1 & 0 & 1 \ldots \ldots \ldots . \\
\bar{y}_{1} & -\bar{x}_{1} & \bar{y}_{2} & -\bar{x}_{2} \ldots \ldots . \\
\bar{x}_{1} & \bar{y}_{1} & \bar{x}_{2} & \bar{y}_{2} \ldots \ldots
\end{array}\right] \\
\bar{x}_{i} & =x_{i}-\frac{1}{p} \sum_{i=1}^{p} x_{i} \\
\bar{y}_{i} & =y_{i}-\frac{1}{p} \sum_{i=1}^{p} y_{i}
\end{aligned}
$$

- In the case of using three dimensional networks, the matrix $S$ has dimensions ( $3 p$ $\mathrm{X7)}$ as follows:
$S^{T}=\left[\begin{array}{ccccccc}1 & 0 & 0 & 1 & 0 & 0 & \ldots \ldots . \\ 0 & 1 & 0 & 0 & 1 & 0 & \ldots \ldots . \\ 0 & 0 & 1 & 0 & 0 & 1 & \ldots \ldots . \\ 0 & \bar{z}_{1} & \bar{y}_{1} & 0 & \bar{z}_{2} & \bar{y}_{2} & \ldots \ldots . \\ -\bar{z}_{1} & 0 & \bar{x}_{1} & -\bar{z}_{2} & 0 & \bar{x}_{2} & \ldots \ldots . \\ \bar{y}_{1} & -\bar{x}_{1} & 0 & \bar{y}_{2} & -\bar{x}_{2} & 0 & \ldots \ldots . \\ \bar{x}_{1} & \bar{y}_{1} & \bar{z}_{1} & \bar{x}_{2} & \bar{y}_{2} & \bar{z}_{2} & \ldots \ldots . .\end{array}\right]$

where:

$$
\bar{z}_{i}=z_{i}-\frac{1}{p} \sum_{i=1}^{p} z_{i}
$$

\section{THE General SOlution OF THE Free DAtUM NETWORK}

Because of the singularity of the matrix $\mathrm{N}$ in the free datum networks a generalized inverse for solution is obtained (e.g Moore-Penrose inverse, [5])

There is an infinite number of solutions that can be obtained for the unknown parameters, $\hat{x}$, to satisfy Eq. (2). This can be expressed in the following form:

$$
\hat{x}=N^{-} U
$$

where $\mathrm{N}$ - is called the generalized inverse of the matrix $\mathrm{N}$ when it satisfies the conditions:

$\mathrm{NN}-\mathrm{N}=\mathrm{N}$ and $\mathrm{N}-\mathrm{NN}-=\mathrm{N}$ -

To solve the problem of the datum defect in free networks, a number of constraints (described previously) are required to define the datum. These constraints must not be less than the number of the datum defects of the matrix $\mathrm{N}$.

In the least squares solution, it is required to minimize the quadratic form of residuals with respect to a given mathematical model and datum constraints [6].

$$
\begin{aligned}
& \text { minimize } \quad \mathrm{v}^{\mathrm{T}} \mathrm{Wv} \\
& f(\bar{x})=\bar{\ell} \\
& \text { subject to } f(\bar{x})=\text { const. or } D^{T} x=c \\
& \Phi=\mathrm{v}^{\mathrm{T}} \mathrm{Wv}^{+}{ }^{2 k_{1}^{T}}(A x-b-v)+2 k_{2}^{T}\left(D^{T} x-c\right) \\
& \frac{\partial \Phi}{\partial v}=2 v^{T} W-2 k_{1}^{T}
\end{aligned}
$$

Transposing and equating to zero

$$
\begin{gathered}
W v-k_{1}=0 \\
k_{1}=W v
\end{gathered}
$$

From the mathematical model of observation equations we have

$v=A x-b$

Using above datum constrains model we have: 


$$
\begin{aligned}
& D^{T} x=c \\
& \frac{\partial \Phi}{\partial x}=2 k_{1}^{T} A+2 k_{2}^{T} D^{T}=0
\end{aligned}
$$

Transposing and equating to zero

$$
A^{T} k+D k_{2}=0
$$

Substitute (21) in (20) to give:

$$
k_{1}=W(A x-b)
$$

Substitute (24) in (23) to give:

$$
\begin{gathered}
A^{T} W(A x-b)+D k_{2}=0 \\
A^{T} W A x-A^{T} W b+D k_{2}=0 \\
\text { or } \quad A^{T} W A x+D k_{2}=A^{T} W b
\end{gathered}
$$

Combining this last equation with Eq. (22), results is given as:

$$
\left[\begin{array}{cc}
A^{T} W A & D \\
D^{T} & O
\end{array}\right]\left[\begin{array}{c}
\hat{x} \\
\hat{k}_{2}
\end{array}\right]=\left[\begin{array}{c}
A^{T} W b \\
c
\end{array}\right]
$$

or

$$
\left[\begin{array}{cc}
N & D \\
D^{T} & 0
\end{array}\right]\left[\begin{array}{l}
\hat{x} \\
\hat{k}
\end{array}\right]\left[\begin{array}{l}
U \\
c
\end{array}\right]
$$

$$
\mathrm{R} \cdot \mathrm{x}=\mathrm{u}
$$

The rank of the matrix $\mathrm{R}$ is equal to its dimensions $(\mathrm{m}+\mathrm{d})$ i.e. it has a full rank; therefore, the inverse of the matrix $\mathrm{R}$ can be obtained directly as follows:

$$
\left[\begin{array}{l}
\hat{x} \\
\hat{k}
\end{array}\right]=\left[\begin{array}{ll}
N & D \\
D^{T} & 0
\end{array}\right]^{-1}\left[\begin{array}{l}
U \\
c
\end{array}\right]
$$

From the well known relation $\mathrm{R}^{-1} \mathrm{R}=\mathrm{RR}^{-1}=\mathrm{I}$ we can write :

$\left[\begin{array}{cc}N & D \\ D^{T} & 0\end{array}\right]\left[\begin{array}{ll}q_{11} & q_{12} \\ q_{21} & q_{22}\end{array}\right]=\left[\begin{array}{cc}I_{m} & 0 \\ 0 & I_{d}\end{array}\right]$

where qij is the sub-matrix of $\mathrm{R}^{-1}$ which is partitioned according to the dimensions of $\mathrm{N}$ and R.

From Eq. (28) the following four basic equations can be established:

$$
\begin{array}{ll}
\mathrm{Nq}_{11}+\mathrm{Dq}_{21} & =\mathrm{Im} \\
\mathrm{Nq}_{12}+\mathrm{Dq}_{22} & =0 \\
\mathrm{D}^{\mathrm{T}} \mathrm{q}_{11} & =0 \\
\mathrm{D}^{\mathrm{T}} \mathrm{q}_{12} & =\mathrm{I}_{\mathrm{d}}
\end{array}
$$

Upper four basic equations are solved to evaluate the elements of the inverse and derived as:

$$
\begin{aligned}
& \mathrm{q}_{22}=0 \\
& \mathrm{q}_{12}=\left(\mathrm{q}_{21}\right)^{\mathrm{T}}=\mathrm{S}\left(\mathrm{D}^{\mathrm{T}} \mathrm{S}\right)^{-1} \\
& \mathrm{q}_{11}=\left(\mathrm{N}+\mathrm{DD}^{\mathrm{T}}\right)^{-1} \mathrm{~N}\left(\mathrm{~N}+\mathrm{DD}^{\mathrm{T}}\right)^{-1}
\end{aligned}
$$

Taking the previous values of the inversion, the general solution of parameters and their covariance matrix using datum constraints $\mathrm{D}^{\mathrm{T}} \mathrm{x}=$ c can be obtained as follows:

$$
\left[\begin{array}{l}
\hat{x} \\
\hat{k}
\end{array}\right]=\left[\begin{array}{ll}
q_{11} & q_{12} \\
q_{21} & q_{22}
\end{array}\right]\left[\begin{array}{l}
U \\
c
\end{array}\right]
$$

The following estimates are obtained:

$$
\begin{aligned}
& \hat{\boldsymbol{x}}=\boldsymbol{q}_{11} \boldsymbol{U}+\boldsymbol{q}_{12} \boldsymbol{c} \\
= & \left(\mathrm{N}+\mathrm{DD}^{\mathrm{T}}\right)^{-1} \mathrm{~N}\left(\mathrm{~N}+\mathrm{DD}^{\mathrm{T}}\right)^{-1} \mathrm{~A}^{\mathrm{T}} \mathrm{Wb}+\mathrm{q}_{12 \mathrm{C}}
\end{aligned}
$$

If $\mathrm{c}=0$ then:

$$
\begin{aligned}
& \hat{x}=q_{11} U=\left(N+D D^{T}\right)^{-1} N\left(N+D D^{T}\right)^{-1} A^{T} W b \\
& \hat{k}=q_{21} U \\
& =\left(\mathrm{S}^{\mathrm{T}} \mathrm{D}\right)^{-1} \mathrm{~S}^{\mathrm{T}} \mathrm{A}^{\mathrm{T}} \mathrm{Wb}
\end{aligned}
$$

but $\mathrm{S}^{\mathrm{T}} \mathrm{A}^{\mathrm{T}}=0$ then

$$
\hat{k}=0
$$

Using the propagation law of errors [3] the covariance matrix of parameters can be obtained from (33) as follows:

$$
\begin{aligned}
& C_{\hat{x}}=q_{11} A^{T} W C_{b} W A q_{11} \\
& \quad=q_{11} A^{T} W W^{-1} W A q_{11} \\
& =q_{11} A^{T} W A q_{11} \\
& C_{\hat{x}}=q_{11} N q_{11}
\end{aligned}
$$

From the definition of the generalized inverse (Grafarend. \& Schaffrin, 1973):

$$
q_{11} N q_{11}=q_{11}
$$

then

$$
\begin{aligned}
& C_{\hat{x}}=q_{11}=\left(N+D D^{T}\right)^{-1} N\left(N+D D^{T}\right)^{-1} \text { or } \\
& C_{\hat{x}}=q_{11}=\left(N+D D^{T}\right)^{-1}-q_{12} q_{21}
\end{aligned}
$$

Many solutions for the parameters $\hat{x}$ and their variances can be obtained using different constraints and different methods for normal matrix inversion. The adjustment of geodetic control networks using least squares models is usually directed to obtain unbiased and precise solution for parameters estimates i.e. the minimization of parameters variances is one of the objectives of this method [6].

Although there are many solutions that can be used in free networks the minimum trace solution is preferred. This solution is identified as the minimum trace solution and gives a minimum trace covariance matrix of parameters i.e. $\sum c_{\hat{x}_{i i}} \Rightarrow$ minimum

Also, it is required to select a set of constants (cn) to minimize the norm of the parameters i.e. the criterion is to be established as $\|\hat{x}\|=\left(\hat{x}^{T} \hat{x}\right)^{1 / 2} \Rightarrow \min$ 


\section{ADJUSTMENTS USING DIFFERENT SOLUTIONS}

Using the leveling network of four stations, different solutions using different datum constraints can be obtained.

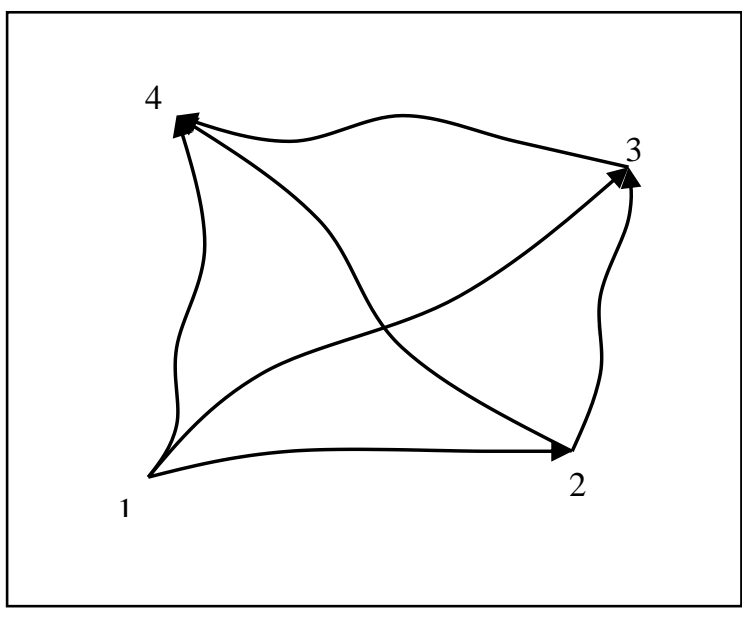

Fig. 1 Vertical Network.

Table 2. - Observations of the Vertical Network

\begin{tabular}{|c|c|c|}
\hline $\begin{array}{c}\text { From } \\
\text { point }\end{array}$ & $\begin{array}{c}\text { To } \\
\text { point }\end{array}$ & Observation(m) \\
\hline 1 & 2 & 1.503 \\
\hline 1 & 3 & 2.005 \\
\hline 1 & 4 & 2.492 \\
\hline 2 & 3 & 0.510 \\
\hline 2 & 4 & 1.002 \\
\hline 3 & 4 & 0.495 \\
\hline
\end{tabular}

From the observation equations and assuming that, observations are taken with the same accuracy, the normal matrix $\mathrm{N}$ is given as:

$$
N=A^{T} W A=\left[\begin{array}{cccc}
3 & -1 & -1 & -1 \\
-1 & 3 & -1 & -1 \\
-1 & -1 & 3 & -1 \\
-1 & -1 & -1 & 3
\end{array}\right]
$$

To obtain the solution for the network we must define the datum using one constraint or fixing the height of one point. Here three types of solutions are obtained using three different datum constraints.

\section{1- Fixed Datum Adjustment}

The matrix $\mathrm{N}$ is a singular matrix means that the regular inverse cannot be obtained unless one reduced level is known. The datum can be defined by fixing the first point. Two approaches for fixed datum solution can be applied:
- Classical Approach(case 1)

The coefficient matrix $\mathrm{A}$ is reduced after eliminating its first column as follows:

$$
\begin{gathered}
A=\left[\begin{array}{ccc}
1 & 0 & 0 \\
0 & 1 & 0 \\
0 & 0 & 1 \\
-1 & 1 & 0 \\
-1 & 0 & 1 \\
0 & -1 & 1
\end{array}\right] \\
N=\left[\begin{array}{ccc}
3 & -1 & -1 \\
-1 & 3 & -1 \\
-1 & -1 & 3
\end{array}\right]
\end{gathered}
$$

and

The inverse of the normal matrix $\mathrm{N}$ can be obtained directly as follows:

$$
N^{-1}=\frac{1}{4}\left[\begin{array}{lll}
2 & 1 & 1 \\
1 & 2 & 1 \\
1 & 1 & 2
\end{array}\right]
$$

Assuming the height of point (1) is equal to zero, then:

$$
A^{T} W b=\left[\begin{array}{c}
-0.009 \\
2.020 \\
3.989
\end{array}\right]
$$

The vector of unknown parameters (heights) and its covariance matrix are computed as:

$$
\begin{aligned}
& \hat{x}_{1}=N^{-1} A^{T} W b=\left[\begin{array}{l}
1.498 \\
2.005 \\
2.497
\end{array}\right] \\
& C_{\hat{x}}=\left[\begin{array}{ccc}
0.5 & 0.25 & 0.25 \\
0.25 & 0.5 & 0.25 \\
0.25 & 0.25 & 0.5
\end{array}\right]
\end{aligned}
$$

and

- Alternative Approach (case 2)

The same solution can be obtained using the following datum constraint with the general solution model:

DTx $=0$

where

$$
D=\left[\begin{array}{l}
1 \\
0 \\
0 \\
0
\end{array}\right]
$$

From the general solution model, using the full matrix $\mathrm{N}$ and matrix $\mathrm{D}$, the following adjustment is obtained:

$$
\begin{aligned}
& C_{\hat{x}}=\left(N+D D^{T}\right)^{-1} N\left(N+D D^{T}\right)^{-1} \\
& \hat{x}=C_{\hat{x}} A^{T} W b \\
& N+D D^{T}=\left[\begin{array}{cccc}
4 & -1 & -1 & -1 \\
-1 & 3 & -1 & -1 \\
-1 & -1 & 3 & -1 \\
-1 & -1 & -1 & 3
\end{array}\right] \\
& \left(N+D D^{T}\right)^{-1}=\frac{1}{4}\left[\begin{array}{cccc}
4 & 4 & 4 & 4 \\
4 & 6 & 5 & 5 \\
4 & 5 & 6 & 5 \\
4 & 5 & 5 & 6
\end{array}\right]
\end{aligned}
$$


$C_{\hat{x}_{1}}=\left[\begin{array}{cccc}0 & 0 & 0 & 0 \\ 0 & 0.5 & 0.25 & 0.25 \\ 0 & 0.25 & 0.5 & 0.25 \\ 0 & 0.25 & 0.25 & 0.5\end{array}\right]$ and $\quad \hat{x}_{1}=\left[\begin{array}{c}0 \\ 1.498 \\ 2.005 \\ 2.497\end{array}\right]$

- The norm of the parameters $\left\|\hat{x}_{1}\right\|=3.53$

- The trace of the covariance matrix $\operatorname{tr} C_{\hat{x}_{1}}=1.5$

\section{2- Free Datum Adjustment}

- Using the full constraints matrix(case 3)

In the following solution the full matrix datum constraints ST $x=0$ is used, then D is substituted by $S$ in the general solution.

$S=\left[\begin{array}{l}1 \\ 1 \\ 1 \\ 1\end{array}\right]$

Using the solution models:

$$
\begin{aligned}
& C_{\hat{x}_{2}}=\left(N+S S^{T}\right)^{-1} N-S\left(S^{T} S\right)^{-1}\left(S^{T} S\right)^{-1} S^{T} \\
& \hat{x}=C_{\hat{x}} A^{T} W b \\
& N+S S^{T}=\left[\begin{array}{llll}
4 & 0 & 0 & 0 \\
0 & 4 & 0 & 0 \\
0 & 0 & 4 & 0 \\
0 & 0 & 0 & 4
\end{array}\right] \\
& \left(N+S S^{T}\right)^{-1}=\frac{1}{4}\left[\begin{array}{cccc}
1 & 0 & 0 & 0 \\
0 & 1 & 0 & 0 \\
0 & 0 & 1 & 0 \\
0 & 0 & 0 & 1
\end{array}\right] \\
& C_{\hat{x}_{2}}=\frac{1}{16}\left[\begin{array}{cccc}
3 & -1 & -1 & -1 \\
-1 & 3 & -1 & -1 \\
-1 & -1 & 3 & -1 \\
-1 & -1 & -1 & 3
\end{array}\right] \\
& \hat{x}_{2}=\left[\begin{array}{c}
-1.500 \\
-0.002 \\
0.505 \\
0.997
\end{array}\right]
\end{aligned}
$$

and

- The norm of the parameters $\left\|\hat{x}_{2}\right\|=1.87$

- The trace of the covariance matrix $\operatorname{tr} C_{\hat{x}_{2}}=0.75$

- Summation of parameters $\sum \hat{x}_{2}=0$

- Partial Free Datum Adjustment(case 4)
In this solution the datum can be defined by using the first three points, point four is removed from datum constraints, as follows:

$$
D^{T} x=0
$$

where:

$$
D=\left[\begin{array}{l}
1 \\
1 \\
1 \\
0
\end{array}\right]
$$

Then, the solution is obtained using the same models in previous sections as follows:

$N+D D^{T}=\left[\begin{array}{cccc}4 & 0 & 0 & -1 \\ 0 & 4 & 0 & -1 \\ 0 & 0 & 4 & -1 \\ -1 & -1 & -1 & 3\end{array}\right]$

$$
\begin{gathered}
C_{\hat{x}_{3}}=\left(N+D D^{T}\right)^{-1} N\left(N+D D^{T}\right)^{-1}=\left[\begin{array}{cccc}
0.167 & -0.083 & -0.083 & 0 \\
-0.083 & 0.167 & -0.083 & 0 \\
-0.083 & -0.083 & 0.167 & 0 \\
0 & 0 & 0 & 0.333
\end{array}\right] \\
\hat{x}_{3}=\left[\begin{array}{c}
-1.168 \\
0.330 \\
0.837 \\
1.329
\end{array}\right]
\end{gathered}
$$

- The norm of the parameters $\left\|\hat{x}_{3}\right\|=1.98$

- The norm of parameters of datum $\left\|\hat{x}_{3}\right\|=1.47$

- The trace of the covariance matrix $\operatorname{tr} C_{\hat{x}_{3}}=0.83$

- Summation of parameters defining the datum $\sum_{i=1}^{3} \hat{x}_{i 3}=0$

\section{RESULTS AND ANALYSIS}

Results obtained from previous adjustments using the four cases of datum constraints, the heights of the four stations and its summations are given. On the other hand the height differences are calculated.

The same parameters and summations are given in case 1 and 2 , in case 3 sum is equal 0 and in case 4 the sum is more than 0 and less than that in the first two cases. All solutions give the same differences in heights. 
Table 3. -Parameters using four Models

\begin{tabular}{|c|c|c|c|c|c|}
\hline station & $\begin{array}{l}\text { case } 1(x \\
1\end{array}$ & $\begin{array}{l}\text { case } 2(x \\
1\end{array}$ & $\begin{array}{l}\text { case3 }(x \\
2\end{array}$ & $\begin{array}{l}\text { case } 4(\times 3 \\
)\end{array}$ & $\begin{array}{l}\text { Height } \\
\text { diff. }\end{array}$ \\
\hline 1 & 0 & 0 & -1.5 & -1.168 & \\
\hline 2 & 1.498 & 1.498 & -0.002 & 0.33 & 1.498 \\
\hline 3 & 2.005 & 2.005 & 0.505 & 0.837 & 0.507 \\
\hline 4 & 2.497 & 2.497 & 0.997 & 1.329 & 0.492 \\
\hline sum & 6 & 6 & 0 & 1.328 & \\
\hline
\end{tabular}

The norm of the parameters and the trace of covariance matrices are calculated from all solutions, and given in Table 4 using the three models.

Table 4. Norm of Parameters and Trace of Covariance Matrix

\begin{tabular}{|l|l|l|l|}
\cline { 2 - 4 } \multicolumn{1}{c|}{} & case 1,2 & case 3 & case 4 \\
\hline $\begin{array}{l}\text { norm of } \\
\text { parameters }\end{array}$ & 3.53 & 1.87 & 1.98 \\
\hline $\begin{array}{l}\text { trace of covariance } \\
\text { matrix }\end{array}$ & 1.5 & 0.75 & 1.47 \\
\hline
\end{tabular}

The minimum norm of adjusted heights and the minimum trace of the covariance matrix are given by the third case when the general solution with full constraints matrix(S) is used.

In Table 5 some solutions obtained from others by adding a scalar calculated from the heights of the first point in the two solution where $\mathrm{k}=\mathrm{x}_{\mathrm{i} 1}-\mathrm{x}_{\mathrm{j} 1}$.

It can be said that the general solution can be transformed to or obtained from any other fixed solution by adding a scalar to all heights. This means that the value of a bench mark can be added to the heights from general solution to give the actual values of heights in the fixed datum solution.

Table 5. Solution from other by adding scalar

\begin{tabular}{|c|c|c|c|c|c|c|}
\hline $\mathrm{x}_{1}$ & $x_{2}$ & $x_{3}$ & $\begin{array}{l}x_{3}=x_{1}+k \\
1\end{array}$ & $\mathrm{x}_{1}=\mathrm{x}_{2}+\mathrm{k}_{2}$ & $\begin{array}{l}x_{3}=x_{2}+k \\
3\end{array}$ & $\begin{array}{l}x_{2}=x_{3}+k \\
4\end{array}$ \\
\hline 0 & -1.5 & -1.168 & -1.168 & 0 & -1.168 & -1.5 \\
\hline $\begin{array}{r}1.49 \\
8\end{array}$ & $\begin{array}{r}-0.00 \\
2\end{array}$ & 0.33 & 0.33 & 1.498 & 0.33 & -0.002 \\
\hline $\begin{array}{r}2.00 \\
5 \\
\end{array}$ & 0.505 & 0.837 & 0.837 & 2.005 & 0.837 & 0.505 \\
\hline $\begin{array}{r}2.49 \\
7 \\
\end{array}$ & 0.997 & 1.329 & 1.329 & 2.497 & 1.329 & 0.997 \\
\hline
\end{tabular}

On the other hand the value of this scalar can be obtained from the mean value of more than one fixed points to obtain the fixed datum solution from the general one.
In Tables 6 and 7 the fixed datum solution $\left(\mathrm{x}_{4}\right)$ with two and three fixed points (B.M1, B.M $M_{2}$ and B.M $\mathrm{M}_{3}$ ) is transformed from the general solution (x) using scalar value calculated from the mean of two and three B.Ms. given the same differences in height and the same observations from others solutions.

Table 6. Fixed datum solution from the general solution using two fixed points

\begin{tabular}{|c|c|c|c|c|c|c|}
\hline point & x(g.solution) & X4(fixed.solution) & $x 4=x+(h 1+h 2) / 2$ & dff from x3 & diff from $x 4$ & \\
\hline 1 & -0.749 & 0 & 0 & & & \\
\hline 2 & 0.749 & 1.498 & 1.498 & 1.498 & 1.498 & \\
\hline 3 & 1.256 & 2.005 & 2.005 & 0.507 & 0.507 & \\
\hline 4 & 1.748 & 2.497 & 2.497 & 0.492 & 0.492 & \\
\hline & B.M1 & $\mathrm{h} 1=0$ & Observations & diff. & diff. from $x 4$ & diff from $x$ \\
\hline & B.M2 & $h 2=1.498$ & 1 to 2 & 1.498 & 1.498 & 1.498 \\
\hline & $(\mathrm{h} 1+\mathrm{h} 2) / 2$ & 0.749 & 1 to 3 & 2.005 & 2.005 & 2.005 \\
\hline & & & 1 to 4 & 2.492 & 2.497 & 2.497 \\
\hline & & & 2 to 3 & 0.51 & 0.507 & 0.507 \\
\hline & & & 2 to 4 & 1.002 & 0.999 & 0.999 \\
\hline & & & 3 to 4 & 0.495 & 0.492 & 0.492 \\
\hline
\end{tabular}

Table 7. Fixed datum solution from the general solution using three fixed points

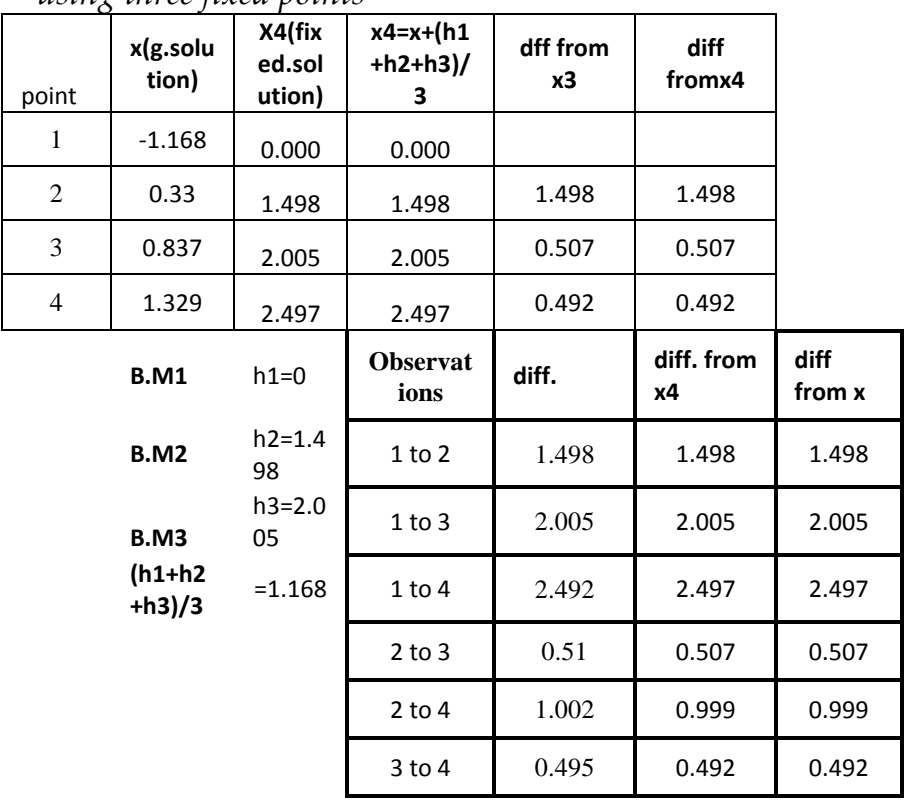

Therefore, the mean obtained from the values of bench marks in fixed datum can be successfully used as scalar to transform fixed solutions from the general solution. 


\section{CONCLUSIONS:}

The same differences of coordinates are given by using any one of the general solutions.

The summation of coordinates is zero when the full constraints datum is used rather than others. The general solution can be used instead of any other fixed classical solution.

The optimal solution is obtained when the minimum trace and the minimum norm solution are given which introduced by using the full constraint matrix $\left(\mathrm{S}^{\mathrm{T}} \mathrm{x}=0\right)$, showing it's suitability for precise survey networks.

A scalar value can be obtained from the height of fixed point or the mean value of more than one fixed points to obtain the fixed datum solution from the general solution.

\section{REFERENCES}

[1] Barnett, R. A. \& Ziegher,M.R.(1987). Applied Calculus, Third Edition, Dellen Publishing Company, Sanfrancisco, California.

[2] Caspary, W. F. (1987). Concepts of Networks and Deformation Analysis, Monograph No.21, the University of New South Wales, School of Surveying.

[3] Cross , P. A. (1983). Advanced Least Squares As Applied to Position-Fixing, Working Paper No.6, University of London.

[4] Fagir, A. H. (1984). Covariance Matrices: Their Structure and Application to the Optimal Design of Geodetic Networks. Ph.D. Thesis. University of N. E. London.

[5] Graarend, E. ESchaffrin, B. (1973). Unbiased Free Net Adjustment, paper at I.A.G. Symposium on Computational Methods in Geometrical Geodesy, Oxford.

[6] Thompson, E. H. (1969). An Introduction to the Algebra of Matrices with Some Applications, Adam Hilger LTD, Twyman House, Bonny Street,London.

[7] G. O. Young, "Synthetic structure of industrial plastics," in Plastics, 2nd ed. vol. 3, J. Peters, Ed. New York: McGraw-Hill, 1964, pp. 15-64 